\title{
Thermodynamics of climate change: generalized sensitivities
}

\author{
V. Lucarini ${ }^{1,2,3}$, K. Fraedrich ${ }^{4}$, and F. Lunkeit ${ }^{4}$ \\ ${ }^{1}$ Department of Meteorology, University of Reading, Earley Gate, P.O. Box 243, Reading RG6 6BB, UK \\ ${ }^{2}$ Department of Mathematics, University of Reading, Whiteknights, P.O. Box 220 Reading RG6 6AX, UK \\ ${ }^{3}$ Walker Institute for Climate System Research, University of Reading, Reading RG6 6AR, UK \\ ${ }^{4}$ Meteorologisches Institut, Klima Campus, University of Hamburg, Grindelberg 5, 20144 Hamburg, Germany
}

Received: 24 December 2009 - Published in Atmos. Chem. Phys. Discuss.: 9 February 2010

Revised: 21 July 2010 - Accepted: 2 October 2010 - Published: 18 October 2010

\begin{abstract}
Using a recent theoretical approach, we study how global warming impacts the thermodynamics of the climate system by performing experiments with a simplified yet Earth-like climate model. The intensity of the Lorenz energy cycle, the Carnot efficiency, the material entropy production, and the degree of irreversibility of the system change monotonically with the $\mathrm{CO}_{2}$ concentration. Moreover, these quantities feature an approximately linear behaviour with respect to the logarithm of the $\mathrm{CO}_{2}$ concentration in a relatively wide range. These generalized sensitivities suggest that the climate becomes less efficient, more irreversible, and features higher entropy production as it becomes warmer, with changes in the latent heat fluxes playing a predominant role. These results may be of help for explaining recent findings obtained with state of the art climate models regarding how increases in $\mathrm{CO}_{2}$ concentration impact the vertical stratification of the tropical and extratropical atmosphere and the position of the storm tracks.
\end{abstract}

\section{Introduction}

The most basic way to characterize the climate system is describing it as a non-equilibrium thermodynamic system, generating entropy by irreversible processes and - if timedependent forcings can be neglected - keeping a steady state by balancing the input and output of energy and entropy with the surrounding environment.

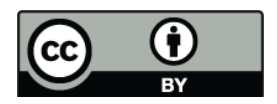

Correspondence to: V. Lucarini (v.lucarini@ reading.ac.uk)
A primary goal of climate science is to understand how the statistical properties of the climate system change as a result of variations in the value of external or internal parameters. Rigorous mathematical foundations to this problem can be traced to the Ruelle response theory for non equilibrium steady state systems (Ruelle, 1998, 2009). Such an approach has been recently proved to have formal analogies with the usual Kubo response theory for quasi-equilibrium systems (Lucarini, 2008a) and to be amenable to numerical investigation (Lucarini, 2009a).

It has long been recognized that a comprehensive view on the climate system can be attained by adopting a thermodynamic perspective. Two main approaches can be envisioned along this line.

In the first approach, the focus is on the dynamical mechanisms and physical processes responsible for the transformation of energy from one form to the other. The concept of the energy cycle of the atmosphere due to Lorenz (1955) allowed for defining an effective climate machine, driven by the temperature difference between a warm and a cold thermal pool. The atmospheric and oceanic motions at the same time result from the mechanical work (then dissipated in a turbulent cascade) produced by the engine, and re-equilibrate the energy balance of the climate system (Peixoto and Oort, 1992). More recently, Johnson (2000) introduced a rather convincing Carnot engine-equivalent picture of the climate by defining robustly the warm and the cold reservoirs and their temperatures.

In the second approach, the emphasis lies on the analysis of the irreversibility of the climate system, and, especially, of its entropy production. This largely results from the intellectual stimulation coming from the maximum entropy production principle (MEPP), which proposes that an

Published by Copernicus Publications on behalf of the European Geosciences Union. 
out-of-equilibrium nonlinear system adjusts in such a way to maximize the production of entropy (Paltridge, 1979). Even if the general validity of MEPP is unclear (Dewar, 2005; Grinstein and Linsker, 2007), its heuristic adoption in climate science has been quite fruitful (Kleidon and Lorenz 2005; Kleidon et al., 2006; Kunz et al., 2008), and has stimulated a detailed re-examination of the importance of entropy production in the climate system (Peixoto and Oort, 1992; Goody, 2000; Ozawa et al., 2003). Moreover, this has resulted into a drive for adopting of a new generation of diagnostic tools based on the 2nd law of thermodynamics for auditing climate models (Fraedrich and Lunkeit, 2008; Pascale et al., 2009; Lucarini et al., 2010) and for outlining a set of parameterisations to be used in conceptual and intermediate complexity models, or for the reconstruction of the past climate conditions (Fraedrich, 2001). Recently a link has been found between the Carnot efficiency, the entropy production and the degree of irreversibility of the climate system (Lucarini, 2009b). This has made possible a new fruitful exploration of the onset and decay of snowball conditions as parametrically controlled by variations in the solar irradiance (Lucarini et al., 2010). In that analysis, the two branches of cold and warm climate stationary states have been found to feature very distinct macro-thermodynamical properties, well beyond the obviously expected large difference in the surface average temperature. The changeovers between icecovered and ice-free planet occur as spontaneous transitions accompanied by a reduction in the efficiency of the climate machine. Moreover, for a given value of the solar constant, the two possible states feature average total material entropy production rates which differ by a factor of about 4 .

In this paper we revise and revive the classic problem of analyzing the climate sensitivity to $\mathrm{CO}_{2}$ concentration changes by adding on top of the usual, IPCC-like (IPCC, 2007) analysis of globally averaged surface temperature changes the investigation of how the global thermodynamics of the system is influenced by the atmospheric composition, so that a wider physically-based set of generalized sensitivities are introduced. Our investigation is performed using the simplified and portable climate model Planet Simulator (PLASIM) (Fraedrich et al., 2005; Fraedrich and Lunkeit, 2008). We believe our work contributes to presenting reliable metrics to be used in the validation of climate models of various degrees of complexity.

\section{Efficiency and entropy production in the climate system}

In this section, for the benefit of the reader, we briefly recapitulate some of the main results recently obtained regarding the description of the non-equilibrium thermodynamical properties of the climate system (Lucarini, 2009a; Lucarini et al., 2010).
We define the total energy of the $\Omega$-domain of the climatic system by $E(\Omega)=P(\Omega)+K(\Omega)$, where $P$ represents the moist static potential energy, given by the thermal - inclusive of the contributions due to water phase transitions - and potential contributions, and $K$ is the total kinetic energy. The time derivative of the total kinetic and potential energy can be expressed as $\dot{K}=-D+W, \dot{P}=\Psi+D-W$, where we have dropped $\Omega$-dependence for convenience, $D$ is the (positive definite) integrated dissipation, $W$ is the instantaneous work performed by the system (or, in other words, the total intensity of the Lorenz energy cycle), $\Psi$ represents the heating due to convergence of heat fluxes (which can be split into the radiative, sensible, and latent heat components), such that $\dot{E}=\Psi$. We denote the total heating rate as $\Phi=\Psi+D$. Under the hypothesis of a non-equilibrium steady state system, we have $\overline{\dot{E}}=\overline{\dot{P}}=\overline{\dot{K}}=0$, where the upper bar indicates time averaging over a long time scale. At any instant, we can partition the domain $\Omega$ into $\Omega^{+}$and $\Omega^{-}$, such that the intensive total heating rate $J$ is positive in $\Omega^{+}$and negative in $\Omega^{-}$:

$\dot{P}+W=\int_{\Omega^{+}} d V \rho J^{+}+\int_{\Omega^{-}} d V \rho J^{-}=\Phi^{+}+\Phi^{-}=\Phi$,

where the upper index refers to the sign of the function, consistently with the definition of the domain of integration.

Since $\bar{D}>0$, we obtain $\bar{W}=\bar{D}=\overline{\dot{\Phi}^{+}}+\overline{\dot{\Phi}^{-}}>0$. Assuming local thermodynamic equilibrium - which applies well everywhere except in the upper atmosphere, which has a negligible mass - and, neglecting the impact of mixing processes (Lucarini, 2009b) we have that locally $\partial s / \partial t=J / T$, where $s$ is the entropy density of the medium. Therefore, at any instant the entropy time derivative has locally the same sign as the heating rate. The time derivative of the total entropy $\dot{S}$ of the system is:

$$
\begin{aligned}
\dot{S} & =\int_{\Omega} d V \rho \partial s / \partial t=\int_{\Omega} d V \frac{\rho J}{T}=\int_{\Omega^{+}} d V \frac{\rho J^{+}}{T}+\int_{\Omega^{-}} d V \frac{\rho J^{-}}{T} \\
& =\int_{\Omega^{+}} d V \rho\left|[\partial s / \partial t]^{+}\right|-\int_{\Omega^{-}} d V \rho\left|[\partial s / \partial t]^{-}\right|=\dot{\Sigma}^{+}+\dot{\Sigma}^{-},(2)
\end{aligned}
$$

where at all times $\dot{\Sigma}^{+}>0$ and $\dot{\Sigma}^{-}<0$. At steady state we have $\overline{\dot{S}}=\overline{\int_{\Omega} d V \rho \partial s / \partial t}=0$, so that no net trend in the total entropy of the system is present, and $\overline{\dot{\Sigma}^{+}}=-\overline{\dot{\Sigma}^{-}}$. Therefore, $\overline{\dot{\Sigma}}^{+}$measures the absolute value of the entropy fluctuations in the unit time integrated throughout the domain, because $2 \overline{\dot{\Sigma}}+=\overline{\int_{\Omega} d V \rho|\partial s / \partial t|}$.

Therefore, we obtain $\overline{\dot{\Sigma}^{+}}=\overline{\Phi^{+}} / \Theta^{+}$and $\overline{\dot{\Sigma}^{-}}=\overline{\Phi^{-}} / \Theta^{-}$, where $\Theta^{+}\left(\Theta^{-}\right)$can be defined as the time and space averaged value of the temperature where absorption (release) of heat occurs. Since $\left|\overline{\dot{\Sigma}^{+}}\right|=\left|\overline{\dot{\Sigma}^{-}}\right|$and $\left|\overline{\Phi^{+}}\right|>\left|\overline{\Phi^{-}}\right|$, we 
derive that $\Theta^{+}>\Theta^{-}$and we characterize the climate system as a Carnot engine such that $\bar{W}=\eta \overline{\Phi^{+}}$, with efficiency $\eta=\left(\Theta^{+}-\Theta^{-}\right) / \Theta^{+}=\left(\overline{\Phi^{+}}+\overline{\Phi^{-}}\right) / \overline{\Phi^{+}}[6,16]$.

The 2nd law of thermodynamics imposes that the longterm average of the material entropy productioninside the system $\overline{\dot{S}_{\text {in }}(\Omega)}$ (this excludes the contributions due to the "degradation" of the solar radiation into terrestrial longwave radiation) is bounded from below by $\overline{\dot{S}}_{\min }(\Omega) \approx \bar{W} /\langle\Theta\rangle \approx$ $\eta \overline{\dot{\Sigma}^{+}}$, where $\langle\Theta\rangle \approx\left(\Theta^{+}+\Theta^{-}\right) / 2$. Therefore, $\eta$ sets also the scale relating the minimal material entropy production of the system to the absolute value of the entropy fluctuation per unit time inside the system. If the system is isothermal and at equilibrium, the internal entropy production is zero, since the efficiency $\eta$ is vanishing: the system has already attained the maximum entropy state. While ${\overline{S_{\text {min }}}}_{\text {is related to the dissipa- }}$ tion of kinetic energy, the excess of material entropy production with respect to the minimum, $\overline{\dot{S}_{\text {exc }}}=\overline{\dot{S}_{\text {in }}}-\overline{\dot{S}_{\text {min }}}$, is due to the heat transport down the gradient of the temperature field. Therefore, we can define:

$$
\begin{aligned}
\alpha & =\overline{\dot{S}_{\mathrm{exc}}} / \overline{\dot{S}_{\min }} \approx \int_{\Omega} d V \bar{H} \cdot \nabla\left(\frac{1}{T}\right) /(\bar{W} /\langle\Theta\rangle) \\
& \approx \int_{\Omega} d V \overline{\boldsymbol{H} \cdot \nabla\left(\frac{1}{T}\right)} /\left(\eta \overline{\dot{\Sigma}^{+}}\right) \geq 0
\end{aligned}
$$

as a parameter of irreversibility of the system (Lucarini, $2009 \mathrm{~b}$ ). The parameter $\alpha$ is basically equivalent to the Bejan number (Paoletti et al., 1989) which measures the irreversibility of the system as the ratio $\overline{\dot{S}}_{\text {in }} /{\overline{S_{\text {min }}}}_{\alpha}=\alpha+1$. When $\alpha=0$ (and the Bejan number is unity), the system features the smallest rate of material entropy production compatible with the presence of a Lorenz energy cycle of intensity $\bar{W}=\bar{D}=\int \overline{\varepsilon^{2}} d V$, as no contributions to entropy production come from fluxes transporting heat down the gradient of the temperature. Since $\overline{\dot{S}_{\text {in }}} \approx \eta \overline{\dot{\Sigma}^{+}}(1+\alpha)$, we have that material entropy production is maximized if we have a joint optimization of heat transport downgradient the temperature field and of production of mechanical work.

\section{Methods}

PLASIM, a simplified yet Earth-like climate model freely available at http://www.mi.uni-hamburg.de/plasim, has been used in a configuration featuring T21 horizontal resolution with five sigma levels in the vertical. The ocean is represented by a $50 \mathrm{~m}$ slab ocean (with energy transport set to 0 ), including a 0-dimensional thermodynamic sea ice model. Slab ocean climate models are well suited for providing an accurate steady state climate response (Danabasoglu and Gent, 2009).
In steady state conditions, the global climate energy budget should vanish when long term averages are considered. If this is not the case, the spurious energy imbalance has to be attributed to unphysical energy sources or sinks. As thoroughly discussed by Lucarini and Ragone (2010), this actually is a serious problem of physical consistency for most state-of-the-art climate models included in 4th Assessment Report of IPCC.

The global atmospheric energy balance is greatly improved with respect to previous versions of the model by adding the kinetic energy losses due to surface friction and horizontal and vertical momentum diffusion (Becker, 2003; Lucarini and Fraedrich, 2009). The average global energy imbalance of the system, computed as long term average of the integrated net radiative flux at the top of the atmosphere, is in all simulations is $\leq 0.2 \mathrm{Wm}^{-2}$, which is about one order of magnitude smaller than most state-ofthe art climate models (Lucarini and Ragone, 2010). The imbalances in the global energy and entropy budgets can be written as $\overline{\dot{S}}=\overline{\dot{\Sigma}^{+}}+\overline{\dot{\Sigma}^{-}}=\Delta_{S}$, with $\Delta_{S} \ll\left|\overline{\dot{\Sigma}^{+}}\right|,\left|\overline{\dot{\Sigma}^{-}}\right|$, and $\dot{\dot{E}}=\overline{\dot{P}}+\overline{\dot{K}}=\Delta_{E}$, with $\Delta_{E} \ll\left|\overline{\Phi^{+}}\right|,\left|\overline{\Phi^{-}}\right|$. Moreover, the Lorenz energy cycle has a spurious term, with $\bar{W}=\overline{\Phi^{+}}+\overline{\Phi^{-}}-\Delta_{E}$. Therefore, the thermodynamic efficiency is ill defined and, similarly, the estimates for entropy production contributions are, in principle, inconsistent. If the numerical errors in the material entropy budget discussed in (Johnson, 2000) are, as in this case, negligible, the 2nd law of thermodynamics imposes that $\Delta_{E} \approx$ $\langle\Theta\rangle \Delta_{S}$. Thanks to that, as thoroughly discussed by Lucarini et al. (2010), the two thermodynamic temperatures $\Theta^{+}$and $\Theta^{-}$are still well defined, as the expression $\left(\Theta^{+}-\Theta^{-}\right) / \Theta^{+}$ provides a good first order approximation to the true efficiency $\left.\eta=\bar{W} / \overline{\Phi^{+}}=\left(\overline{\Phi^{+}}+\overline{\Phi^{-}}-\Delta_{E}\right) / \overline{\Phi^{+}}\right)$. Similarly, the material entropy production rate is computed as $\overline{\dot{S}_{\text {in }}}+\Delta_{S}$, and the irreversibility factor is evaluated as $\alpha=$ $\left(\dot{\bar{S}}_{\text {in }}+\Delta_{S}\right) /\left(\eta\left(\dot{\bar{\Sigma}}+^{+}+\left|\dot{\bar{\Sigma}}^{-}\right|\right) / 2\right)$, where we introduce a correction in the denominator to account for the fact that $\dot{\bar{\Sigma}}^{+} \neq\left|\dot{\bar{\Sigma}}^{-}\right|$. With these corrections, all proposed formulas apply with a high degree of approximation.

\section{Results}

In the usual operative definition, climate sensitivity $\Lambda_{T_{S}}$ is the increase of the globally averaged mean surface temperature $\overline{T_{S}}$ between the preindustrial $\mathrm{CO}_{2}$ concentration steady state and the steady state conditions realized when $\mathrm{CO}_{2}$ concentration is doubled. As $\overline{T_{S}}$ is almost linear with respect to the logarithm of the $\mathrm{CO}_{2}$ concentration on a large range, it is actually easy to generalize the definition of $\Lambda_{T_{S}}$ as the impact on $\overline{T_{S}}$ of $\mathrm{CO}_{2}$ doubling so that $\Lambda_{T_{S}}=d \overline{T_{S}} / d \log _{2}\left(\left[\mathrm{CO}_{2}\right]_{\mathrm{ppm}}\right)$. Such a linear behaviour basically results from the fact that up to a good degree of approximation, the $\mathrm{CO}_{2}$-related radiative 


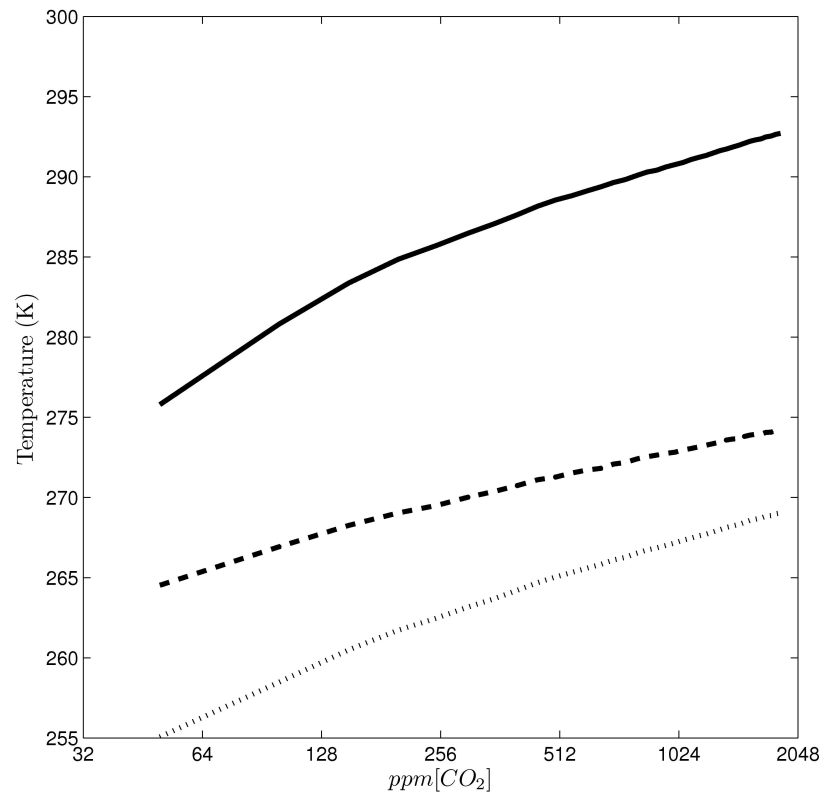

Fig. 1. Time average of the global mean surface temperature $T_{S}$ (solid line) and of the temperature of the warm $\left(\Theta^{+}\right)$and cold $\left(\Theta^{-}\right)$ reservoirs (dashed and dotted lines, respectively).

forcing is linear with the logarithm of the $\mathrm{CO}_{2}$ concentration (Myhre et al., 1998; IPCC, 2007).

The main goal of this work is to check whether it is possible to define, in a similar fashion, generalized sensitivities $\Lambda_{X}$ to describe the steady state response to $\mathrm{CO}_{2}$ concentration changes of the thermodynamical properties $X$ of the climate system. At this scope, we have performed climate simulations for $\mathrm{CO}_{2}$ concentrations ranging from 50 to $1850 \mathrm{ppm}$ by $50 \mathrm{ppm}$ steps, thus totalling 37 runs. Each simulation has a length of 50 years and the statistics are computed on the last 30 years of the simulations in order to rule out the presence of transient effects. In order to fully characterize the non-equilibrium properties of the climate system, we have analysed the most important thermodynamic variables of the system introduced in the previous section:

- the time average of the temperatures of the warm $\left(\Theta^{+}\right)$ and cold $\left(\Theta^{-}\right)$reservoir, and, as reference, the time average of the global mean surface temperature $\overline{T_{S}}$ (Fig. 1);

- the thermodynamic efficiency $\eta$ (Fig. 2a);

- the average intensity of the Lorenz energy cycle $\bar{W}$ (Fig. 2b);

- the time average of the rate of material entropy production $\overline{\dot{S}_{\text {in }}}$ (Fig. 2c);

- the degree of irreversibility of the system $\alpha$ (Fig. 2d).
Table 1. Generalized Sensitivities.

\begin{tabular}{lr}
\hline Definition & Value \\
\hline$\Lambda_{\overline{T_{S}}}$ & $2.55 \mathrm{~K}^{\mathrm{a}}$ \\
$\Lambda_{\Theta^{+}}$ & $1.65 \mathrm{~K}$ \\
$\Lambda_{\Theta^{-}}$ & $2.35 \mathrm{~K}$ \\
$\Lambda_{\eta}$ & -0.002 \\
$\Lambda_{\overline{\dot{W}}}$ & $-0.06 \mathrm{Wm}^{-2}$ \\
$\Lambda_{\overline{\dot{S}_{\text {in }}}}$ & $0.0004 \mathrm{Wm}^{-2} \mathrm{~K}^{-1}$ \\
$\Lambda_{\alpha}$ & 0.7 \\
\hline
\end{tabular}

${ }^{a}$ The value refers to $\mathrm{CO}_{2}$ concentrations larger than $150 \mathrm{ppm}$.

In addition to the surface temperature, all of these thermodynamic variables feature a monotonic behaviour with respect to the $\mathrm{CO}_{2}$ concentration. In particular, all of the variables feature an approximate linear behaviour with respect to the logarithm of the $\mathrm{CO}_{2}$ concentration. Therefore, we can safely attribute a robust value (with an uncertainty of at most $10 \%$ ) to the generalized sensitivities defined as $\Lambda_{X} \equiv d X / d \log _{2}\left(\left[\mathrm{CO}_{2}\right]_{\mathrm{ppm}}\right)$. Results are summarized in Table 1 . Note that the variable with the strongest systematic deviations from linearity is the globally averaged surface temperature, which features a markedly linear dependence only for $\mathrm{CO}_{2}$ concentrations larger than $150 \mathrm{ppm}$. Instead, the graph of the rate of material entropy production is noisier than the other variables, but linearity with respect to the logarithm of $\mathrm{CO}_{2}$ concentration is on the average quite accurate.

The three temperature indicators (Fig. 1) feature, as expected, positive sensitivities: the surface temperature sensitivity (evaluated in the, more realistic, upper range of $\mathrm{CO}_{2}$ concentrations) is well within the range of what is simulated by the climate models included in 4th IPCC report (IPCC, 2007), whereas the two bulk thermodynamic temperatures have smaller sensitivities. In order to interpret these results, we consider the changes in the heating and temperature patterns between the 1000 and $100 \mathrm{ppm} \mathrm{CO}_{2}$ concentration runs. In the $100 \mathrm{ppm} \mathrm{CO}_{2}$ concentration run, on the average, positive heating patterns (which are relevant for defining $\Theta^{+}$) include the whole equatorial troposphere and the lower levels (below $700 \mathrm{hPa}$ ) in the wide latitudinal belt between the Northern and Southern Mid-latitudes (Fig. 3a). When looking at the vertically integrated patterns (Fig. 4a), we discover a major zonal asymmetry, namely that in the mid-latitudes the air aloft the continental masses experiences negative heating rates (as opposed to the ocean areas), thus suggesting that over land the low level positive heating pattern is shallower and/or weaker. In Fig. $3 \mathrm{~b}$ it is shown that, in the $1000 \mathrm{ppm}$ $\mathrm{CO}_{2}$ concentration run, the extent of the zonally averaged positive heating region is wider than in the $100 \mathrm{ppm}$ case, as it includes higher latitudes and lower pressure portions of the atmosphere. Looking at the vertically integrated patterns (Fig. 4b), we consistently find a northward shift of the 
a)

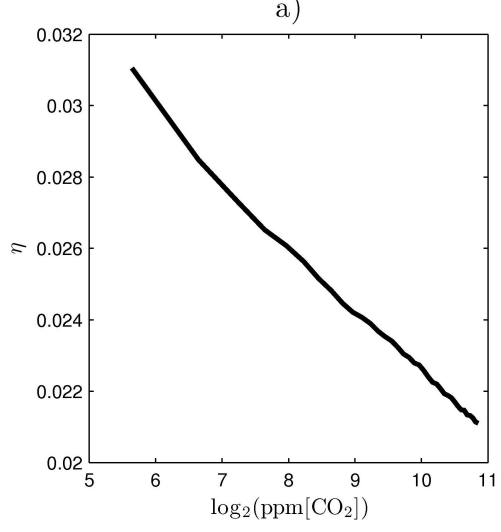

c)

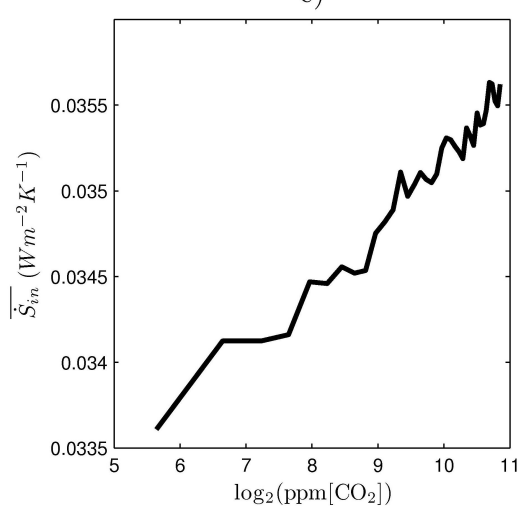

b)

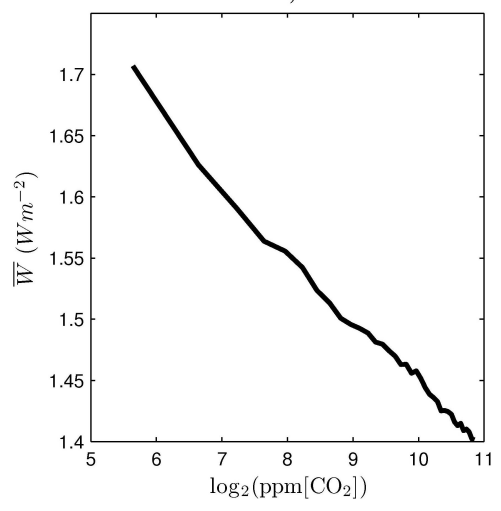

d)

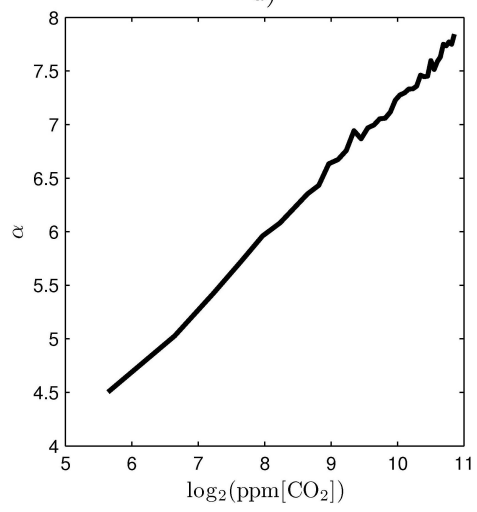

Fig. 2. Generalised climate sensitivities. $\mathrm{CO}_{2}$ concentration dependence of macroscopic thermodynamic variables: (a) efficiency; (b) intensity of the Lorenz energy cycle (or, equivalently, total dissipation); (c) material entropy production; (d) degree of irreversibility. See text for details.

positive heating regions in the mid-latitudes. This is closely related to the poleward migration of the storm track and of the peak of meridional enthalpy transport in both hemispheres under warmer climate conditions, which has been carefully described in state-of-the-art climate models (Yin, 2005; Lucarini and Ragone, 2010). Instead, the air aloft the continental masses experiences negative heating patterns on the average as in the $100 \mathrm{ppm} \mathrm{CO}_{2}$ concentration case. The fact that relatively colder regions of the atmosphere are considered in the integrals leading to the definition of $\Theta^{+}$for increasing values of $\mathrm{CO}_{2}$ concentrations seems to explain why the sensitivity of $\Theta^{+}$is smaller than that of $\Theta^{-}$.

The reason why the sensitivity of $\Theta^{-}$is smaller than that of the surface temperature can be ascertained by looking at the actual patterns of average temperature change between the two runs (Fig. 5a). We observe that the most of the region with negative net heating balances - which concurs to defining $\Theta^{-}$- experiences a larger increase of the vertical temperature gradient, as temperature increases aloft are smaller than those close to surface. Therefore, its average temperature will increase less than the average surface temperature for increasing $\mathrm{CO}_{2}$ concentrations.
We observe that, whereas the mid-latitude atmosphere becomes more stable, in agreement with what observed in more realistic climate models (Frierson, 2006), the equatorial region the situation is reversed, as the largest temperature increases are observed in the upper troposphere, since the large increase in water vapour content manages to decrease substantially the moist adiabatic lapse rate. Also the latter result is in agreement with what found in state-of-the-art climate models (Chou et al., 2009).

We can rephrase the fact that with higher $\mathrm{CO}_{2}$ concentrations the temperature of the cold bath increases faster than that of the warm bath by saying that on the average we have a more isothermal atmosphere. In fact, by looking at Fig. 5a, we find that the atmospheric region with the highest average temperature (the tropical lower troposphere) features the smallest temperature increase, whereas large temperature increases are observed in cold regions such as the lower polar troposphere and the whole upper troposphere. The main driver of the reduction of the planetary thermal differences is the large enhancement of the convergence of latent heat with increasing $\mathrm{CO}_{2}$ concentration. The pattern of change in the zonally averaged field of latent heat heating between the 

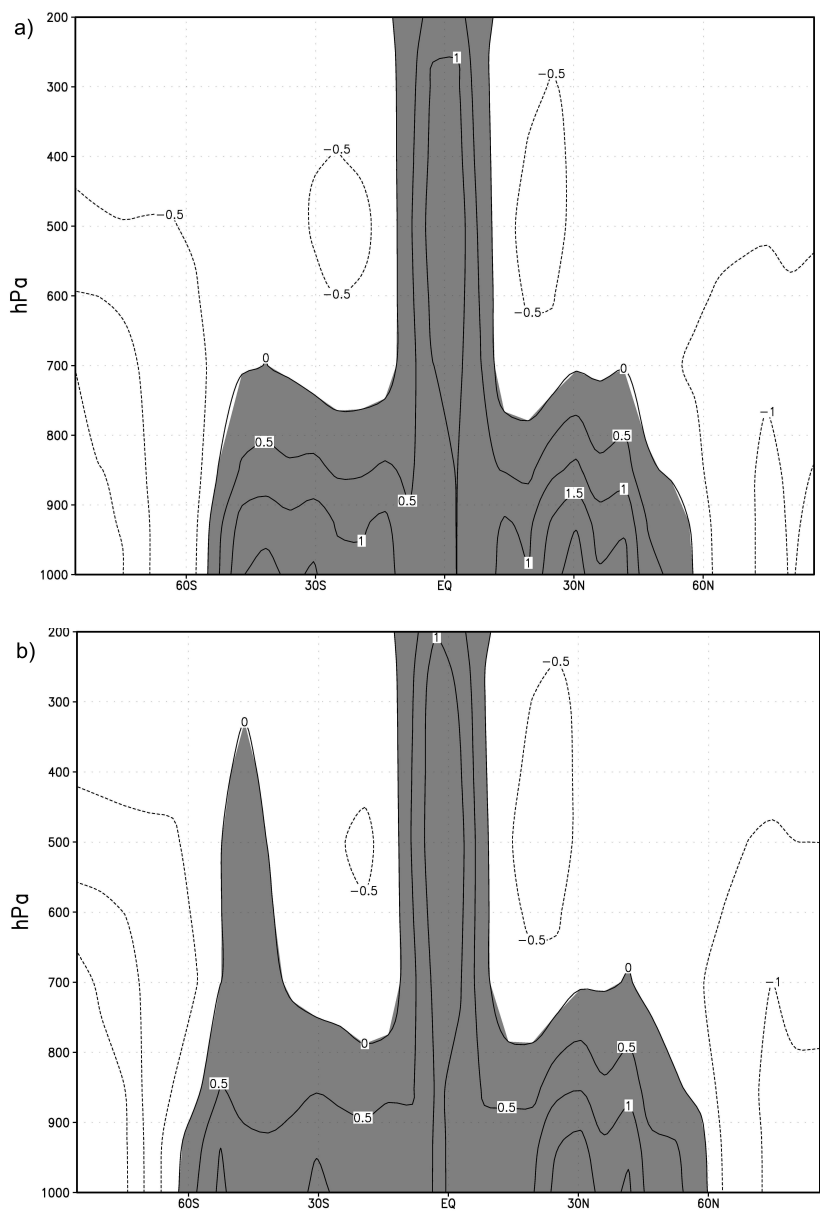

Fig. 3. Zonally averaged mean heating rates (in $\mathrm{K} /$ day) for the 100 (a) and 1000 (b) ppm $\mathrm{CO}_{2}$ concentration runs.

1000 and $100 \mathrm{ppm} \mathrm{CO}_{2}$ concentration runs resembles closely that of mean temperature change depicted in Fig. 5a.

Consequently, increases in the $\mathrm{CO}_{2}$ concentration cause a steep decrease in the efficiency of the climate system, as shown in Fig. 2a. In the explored range, the efficiency decreases by about $35 \%$, with a relative change of about $-7 \%$ per $\mathrm{CO}_{2}$ doubling. In a thicker (and warmer) atmosphere, the total absorbed heat $\overline{\Phi^{+}}$is larger, so that the actual strength of the Lorenz energy cycle changes as the result of the competing effects of increasing energy input and decreasing efficiency. The intensity of the Lorenz cycle decreases in a warmer climate (Fig. 2b), with an approximate change of $-4 \%$ per $\mathrm{CO}_{2}$ doubling. By energy conservation, the same applies to the total dissipation, so that in a warmer climate weaker surface winds are expected, as found by HernandezDeckers and Von Storch (2010) is a recent paper. This is confirmed by inspecting Fig. 6a, where changes in the average square velocity in the lowest sigma level of the model are reported between the 1000 and the $100 \mathrm{ppm} \mathrm{CO}_{2}$ concentration runs. Interestingly, while changes are small over most latitudes, the negative contributions are concentrated in
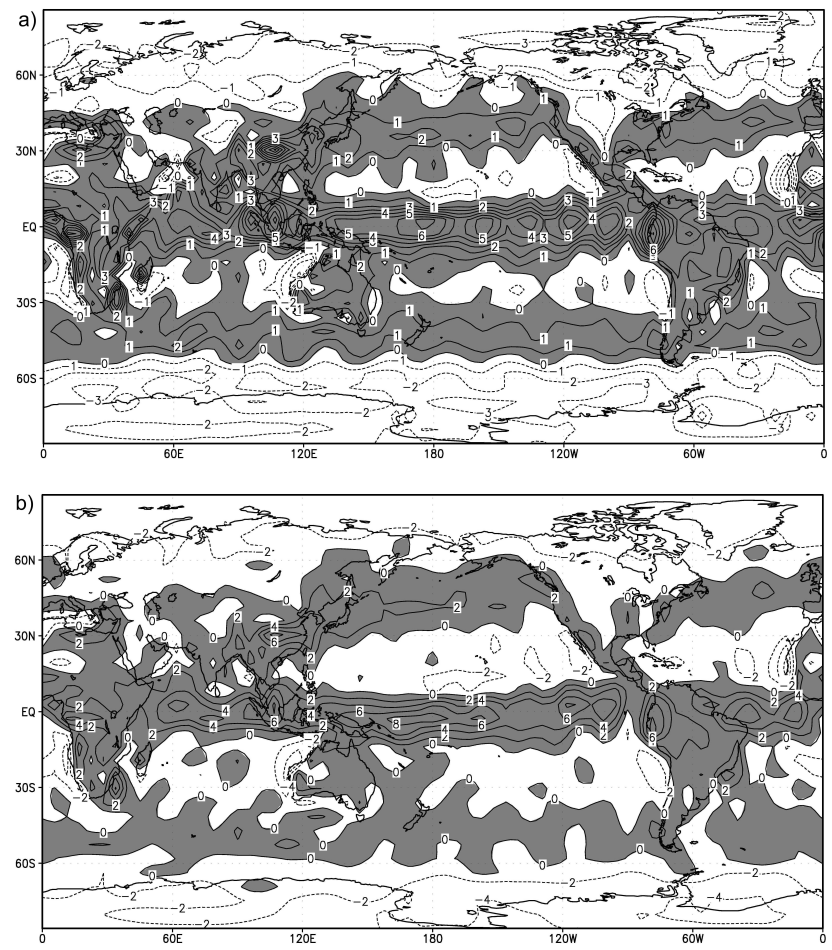

Fig. 4. Vertically averaged mean heating rates (in $\mathrm{K} /$ day) for the 100 (a) and 1000 (b) ppm $\mathrm{CO}_{2}$ concentration runs.

the mid-to-high latitudes of the Southern Hemisphere. Note that the pattern shown in Fig. 6a resembles qualitatively the difference between the 350 and the $100 \mathrm{ppm} \mathrm{CO}_{2}$ concentration runs, whereas, when observing the changes between the $1000 \mathrm{ppm}$ and the $350 \mathrm{ppm}$ concentration runs (Fig. 6b), we have the negative contributions between $40^{\circ}$ and $50^{\circ} \mathrm{S}$ to be partially compensated by a positive change in the latitudinal belt $50^{\circ} \mathrm{S}-60^{\circ} \mathrm{S}$, in qualitative agreement with what shown, e.g. by Kushner et al. (2001) in a typical global warming experiment. This latter feature suggests that PLASIM, in spite of its simplifications and the absence of a coupled dynamic ocean, is able to capture the well known trend of strengthening of the wind stress in the region of the Antarctic Circumpolar Current in a warmer climate. The discrepancies between the zonal profiles depicted in Fig. $6 \mathrm{a}$ and Fig. $6 \mathrm{~b}$ suggest that, latitude by latitude, the changes is the dissipation are not linear (or even monotonic) with the logarithm of the $\mathrm{CO}_{2}$ concentration. This is not in contradiction with the approximately linear dependence found for the total dissipation (or, equivalently, for intensity of the global Lorenz energy cycle) and depicted in Fig. 2b. Locally, there is no guarantee of linearity, since climate patterns change in a complex fashion as $\mathrm{CO}_{2}$ concentration is altered.

As with increasing $\mathrm{CO}_{2}$ concentration the average temperature increases and the total dissipation decreases, the quantity $\overline{\dot{S}}_{\min }$, which is uniquely related to mechanical dissipation, must be a decreasing function of $\mathrm{CO}_{2}$ concentration. 
a)
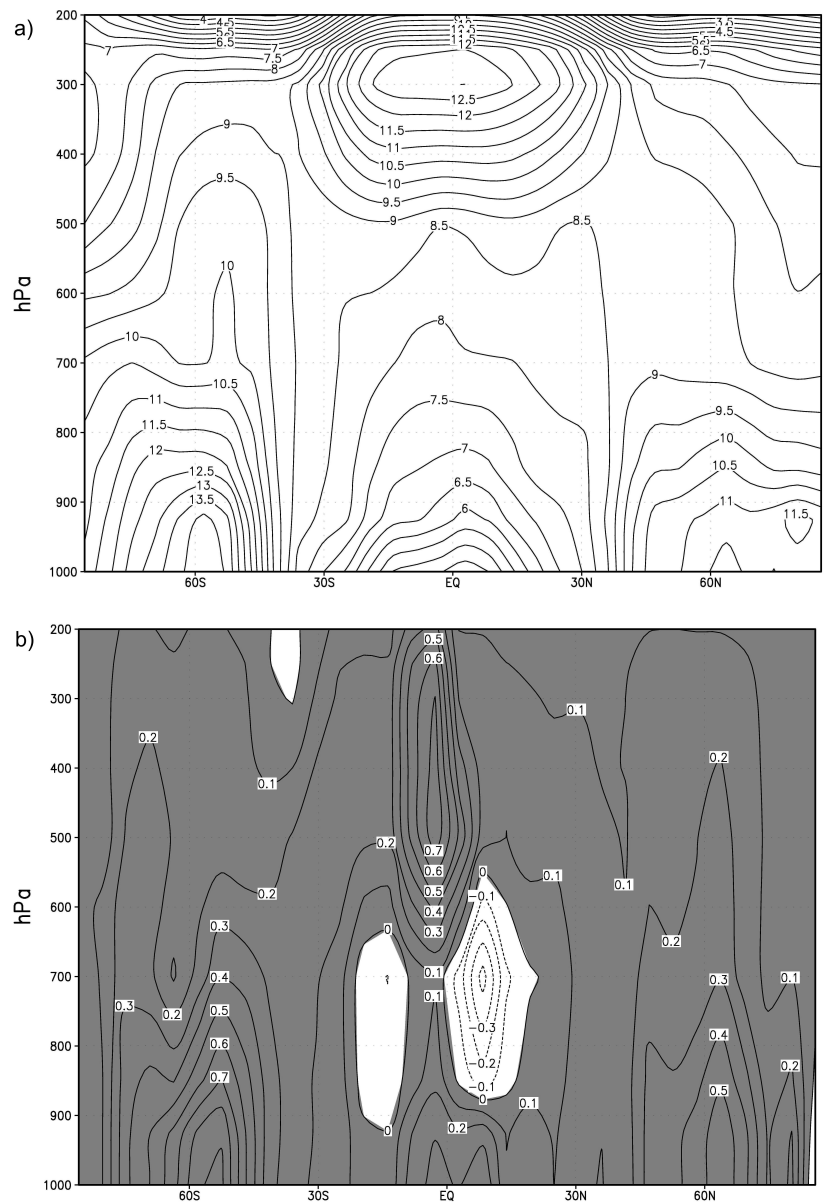

Fig. 5. (a) Zonally averaged mean temperature difference between the $1000 \mathrm{ppm} \mathrm{CO}$ and $100 \mathrm{ppm} \mathrm{CO}_{2}$ concentration runs. (b) Zonally averaged difference between the $1000 \mathrm{ppm} \mathrm{CO}_{2}$ and $100 \mathrm{ppm}$ $\mathrm{CO}_{2}$ concentration runs for the mean heating rates due to the convergence of latent heat fluxes (in K/day).

Instead, as shown in Fig. 2c, the actual average total rate of material entropy production $\overline{\dot{S}_{\text {in }}}=\overline{\dot{S}_{\text {min }}}+\overline{\dot{S}_{\text {exc }}}$ has the opposite behaviour, with an approximate relative increase of $2 \%$ per $\mathrm{CO}_{2}$ doubling. This implies that $\overline{\dot{S}_{\text {exc }}}$, giving the entropy production due to the heat transport down the gradient of the temperature field, is much higher in warmer climates, the reason being that the convergence of latent heat fluxes becomes extremely effective in transporting heat from warm to cold temperature regions (Fig. 5b). Therefore, the degree of irreversibility of the system $\alpha$ increases steeply with $\mathrm{CO}_{2}$ concentration (Fig. 2d). In the considered range, the fraction of entropy production due to mechanical energy dissipation $1 /(\alpha+1)$ drops from about $22 \%$ to about $12 \%$. This behaviour is specific for climate conditions analogous to the present ones, whereas under snowball conditions, where latent heat fluxes are negligible, the value of $\alpha$ is about unity and only slightly affected by temperature (Lucarini et al., 2010).
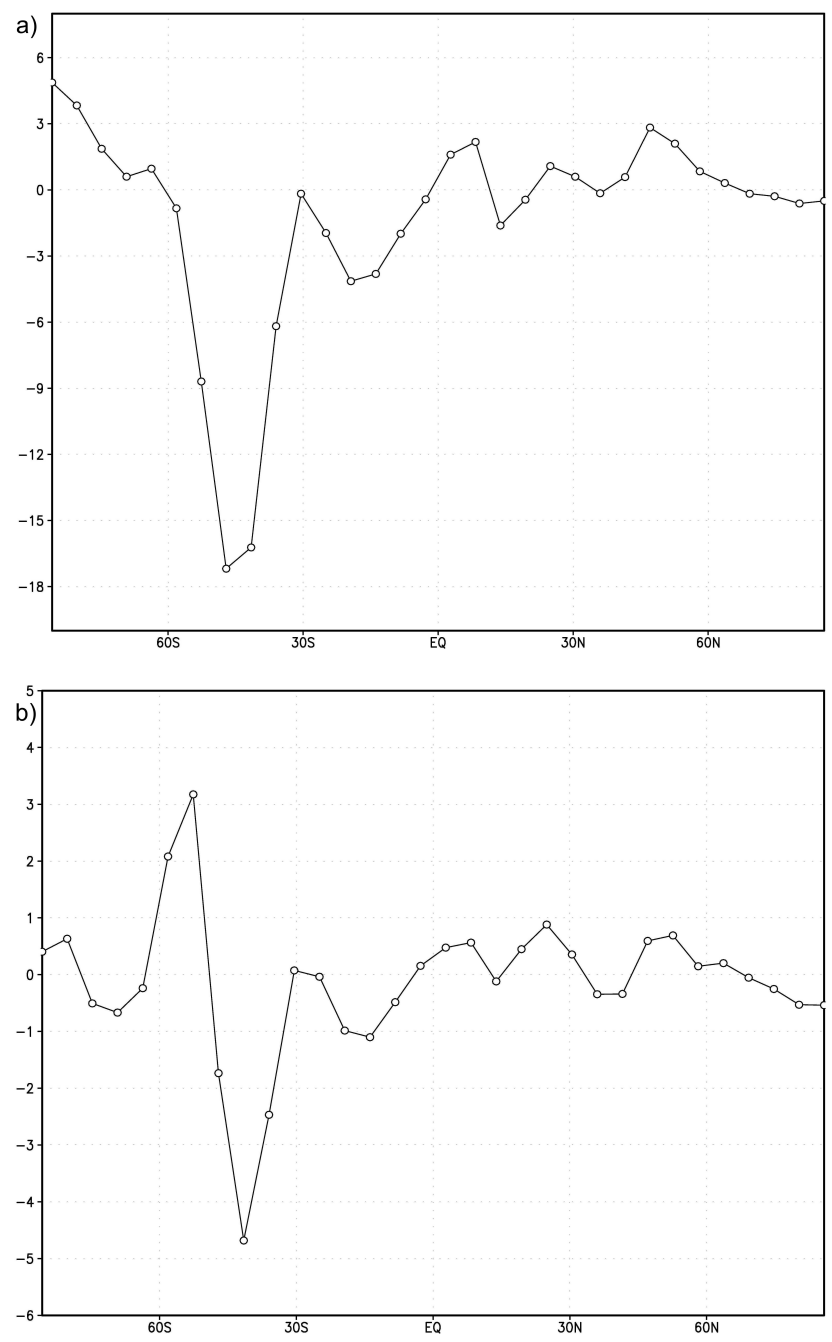

Fig. 6. (a) Zonally averaged difference between the $1000 \mathrm{ppm} \mathrm{CO}_{2}$ and $100 \mathrm{ppm} \mathrm{CO}_{2}$ runs for the mean squared velocity (in $\mathrm{m}^{2} \mathrm{~s}^{-2}$ ) at the sigma level closest to surface. (b) Same as (a), but the difference between the $1000 \mathrm{ppm} \mathrm{CO}_{2}$ and $350 \mathrm{ppm} \mathrm{CO}_{2}$ runs is shown.

\section{Conclusions}

Using a simplified yet Earth-like climate model, we have reexamined the classical problem of the steady-state response of the climate system to $\mathrm{CO}_{2}$ concentration variations and have focused on the changes in the non-equilibrium thermodynamical properties of the climate system, building upon a recently introduced theoretical framework (Lucarini, 2009b). The novel approach presented here allows for setting in common framework the changes global properties of the climate system such as the intensity of the Lorenz energy cycle and the material entropy production. As a result, we have introduced a comprehensive set of generalized climate sensitivities. 
Table 2. Parameterisations ${ }^{\mathrm{a}}$.

\begin{tabular}{lr}
\hline Definition & Value \\
\hline$d \Theta_{1} / d \overline{T_{s}}$ & 0.65 \\
$d \Theta_{2} / d \overline{T_{s}}$ & 0.92 \\
$d \eta / d \overline{T_{s}}$ & $-0.0008 \mathrm{~K}^{-1}$ \\
$d \dot{\bar{W}} / d \overline{T_{s}}$ & $-0.024 \mathrm{Wm}^{-2} \mathrm{~K}^{-1}$ \\
$d \dot{\dot{S}_{\text {in }}} / d \overline{T_{S}}$ & $0.00016 \mathrm{Wm}^{-2} \mathrm{~K}^{-2}$ \\
$d \alpha / d \overline{T_{s}}$ & $0.275 \mathrm{~K}^{-1}$ \\
\hline
\end{tabular}

a Values are valid for .globally averaged surface temperatures higher than $284 \mathrm{~K}$.

In addition to the average surface temperature, the intensity of the Lorenz energy cycle, the Carnot efficiency, the material entropy production and the degree of irreversibility of the system change monotonically with the $\mathrm{CO}_{2}$ concentration. The behaviour is approximately linear with the logarithm of the $\mathrm{CO}_{2}$ concentration, basically as a result of the fact that the net radiative forcing exerted by $\mathrm{CO}_{2}$ has an analogous dependence.

It is rather interesting to observe that, among the considered variables, the largest systematic deviations from linearity (relevant for values of the $\mathrm{CO}_{2}$ concentration smaller than $150 \mathrm{ppm}$ ) are observed for the globally averaged surface temperature, which is, somewhat ironically, the quantity that motivated the introduction of the climate sensitivity. This is actually not so surprising because, whereas the other thermodynamical quantities describe bulk properties of the atmosphere which are directly impacted by the changes in the $\mathrm{CO}_{2}$-related radiative forcing, the surface temperature is more of a boundary property.

The resulting generalized sensitivities proposed here (whose values are reported in Table 1) demonstrate that the climate system becomes less efficient (and more isothermal), more irreversible, and features higher entropy production as it becomes warmer. Changes in the intensity of the latent heat fluxes are the dominating ingredients, thus showing, at a fundamental level, how important it is to address correctly the impact of climate change on the hydrological cycle.

Thanks to the monotonic dependence (and not, specifically, to the linearity) of the diagnosed variables with respect to the logarithm of the $\mathrm{CO}_{2}$ concentration, it is possible to reparameterise efficiently all the variables with respect to just one. As an example of this procedure, in Table 2 we provide the linear coefficients of all the thermodynamic macrovariables of the system with respect to the average surface temperature. These coefficients are valid for $\mathrm{CO}_{2}$ concentrations larger than $150 \mathrm{ppm}$ and, correspondingly, for globally averaged surface temperatures larger than $284 \mathrm{~K}$. These data may be of use when devising simplified yet comprehensive climate models or estimating unknown quantities in comprehensive models or from observational data.
We believe that the investigation proposed here may stimulate the re-examination, from a more fundamental point of view, of the problem of climate change, for studying the impacts of changing the atmospheric composition on the properties of the large scale atmospheric circulation, of convection, and of the processes responsible for coupling the atmosphere with land and ocean.

In this study it is shown that the heating patterns, which are crucial for constructing all of the non-equilibrium thermodynamical properties of the system discussed here, respond to increasing $\mathrm{CO}_{2}$ concentration consistently with how state of the art climate models describe changes in the stratification in the tropical (Chou et al., 2006) and extratropical atmosphere (Frierson, 2006) as well as in the properties of the meridional atmospheric enthalpy transport (Yin, 2005; Lucarini and Ragone, 2010).

Following the results an earlier study on the onset and the decay of snowball conditions (Lucarini et al., 2010), we propose that the approach presented here may be of help for addressing problems of paleoclimatological relevance, such as the interplay between solar irradiance and atmospheric composition variations in determining slow climate modulations as well as tipping points (Lenton et al., 2008), as, e.g., the onset and decay of ice ages or rapid changes in the large scale properties of the ocean circulation.

The present study calls for several improvements. Firstly, it would be important to address the role of a dynamic ocean, which in the present analysis acts an infinite source of water and is relevant for increasing the thermal inertia of the system. Secondly, it would be very interesting to treat timedependent changes in the $\mathrm{CO}_{2}$ concentration and study the adjustment process of the system in terms of the proposed global thermodynamical quantities.

We expect that extensive application of the thermodynamically-based tools adopted here may, in general, help closing the Gap between Simulation and Understanding in Climate Modeling (Held, 2005) and may provide the basis for a new generation of metrics aimed at the validation of climate models (Lucarini, 2008b).

Acknowledgements. The referees' and editor's comments are appreciated. KF acknowledges the support through the Max-Planck Fellow Group. VL acknowledges the support by the European Commission through the FP7-ERC Starting Grant project NAMASTE (Grant Agreement number: 257106).

Edited by: P. Haynes

\section{References}

Becker, E., Frictional heating in global climate models, Mon. Weather Rev., 131, 508-520, 2003.

Chou, C., Neelin, J. D., Chen, C.-A., and Tu, J.-Y.: Evaluating the "Rich-Get-Richer" Mechanism in Tropical Precipitation Change under Global Warming, J. Climate, 22, 1982-2005, 2009. 
Danabasoglu, G. and Gent, P. R.: Equilibrium Climate Sensitivity: is it accurate to use a slab ocean model?, J. Climate, 22, 24942499, 2009.

Dewar, R.: Maximum entropy production and the fluctuation theorem, J. Phys. A: Math. Gen., 38, L371-L381, 2005.

Fraedrich, K.: Simple Climate Models, in: Progress in Probability 49, edited by: Imkeller, P. and von Storch, J.-S., Birkhäuser, Berlin, 65-110, 2001.

Fraedrich, K. and Lunkeit, F.: Diagnosing the entropy budget of a climate model, Tellus A, 60, 921-931, 2008.

Fraedrich, K., Jansen, H., Kirk, E., Luksch, U., and Lunkeit, F.: The planet simulator: towards a user friendly model, Meteorol. Zeitschrift, 14, 299-304, 2005.

Frierson D. M. W.: Robust increases in midlatitude static stability in simulations of global warming, Geophys. Res. Lett., 33, L24816, doi:10.1029/2006GL027504, 2006.

Grinstein G. and Linsker R.: Comments on a derivation and application of the 'maximum entropy production' principle, J. Phys. A: Math. Theor., 40, 9717-9720, 2007.

Held, I. M.: The Gap between Simulation and Understanding in Climate Modeling, Bull. Amer. Meteor. Soc., 86, 1609-1614, 2005.

Hernández-Deckers, D. and von Storch, J.-S.: Energetics Responses to Increases in Greenhouse Gas Concentration, J. Climate, 23, 3874-3887, doi:10.1175/2010JCLI3176.1, 2010.

IPCC: The Physical Science Basis. Contribution of Working Group I to the Fourth Assessment Report of the Intergovernmental Panel on Climate Change, Cambridge University Press, Cambridge, 2007.

Johnson D. R.: Entropy, the Lorenz Energy Cycle and Climate, in: General Circulation Model Development: Past, Present and Future, edited by: Randall, D. A., Academic Press, New York, 659$720,2000$.

Kleidon, A. and Lorenz, R. D. (eds.): Non-equilibrium thermodynamics and the production of entropy: life, Earth, and beyond, Springer, Berlin, 2005.

Kleidon, A., Fraedrich, K., Kirk, E., and Lunkeit, F.: Maximum entropy production and the strength of boundary layer exchange in an atmospheric general circulation model, Geophys. Res. Lett., 33, L06706, doi:10.1029/2005GL025373, 2006.

Kunz, T., Fraedrich, K., and Kirk, E.: Optimisation of simplified GCMs using circulation indices and maximum entropy production, Clim. Dyn., 30, 803-813, 2008.

Kushner, P. J., Held, I., and Delworth, T. W.: Southern Hemisphere atmospheric circulation response to global warming, J. Climate, 14, 2238-2249, 2001.

Lenton, T. M., Held, H., Kriegler, E., Hall, J. W., Lucht, W., Rahmstorf, S., and Schellnhuber, H. J.: Tipping elements in the Earth's climate system, P. Natl. Acad. Sci., 105, 1786-1793, 2008.

Lorenz, E. N.: Available potential energy and the maintenance of the general circulation, Tellus, 7, 157-167, 1955.
Lucarini, V.: Response Theory for Equilibrium and NonEquilibrium Statistical Mechanics: Causality and Generalized Kramers-Kronig relations, J. Stat. Phys., 131, 543-558, 2008a.

Lucarini, V.: Validation of Climate Models, in: Encyclopedia of Global Warming and Climate Change, edited by: Philander, SAGE, Thousand Oaks, 1053-1057, 2008b.

Lucarini, V.: Evidence of dispersion relations for the nonlinear response of the Lorenz 63 system, J. Stat. Phys., 134, 381-400, 2009a.

Lucarini, V.: Thermodynamic Efficiency and Entropy Production in the Climate System, Phys Rev. E, 80, 021118, doi:10.1103/PhysRevE.80.021118, 2009b.

Lucarini, V. and Fraedrich K.: Symmetry breaking, mixing, instability, and low frequency variability in a minimal Lorenz-like system, Phys. Rev. E, 80, 026313, doi:10.1103/PhysRevE.80.026313, 2009.

Lucarini, V. and Ragone, F.: Energetics of PCMDI/CMIP3 Climate Models: Net Energy Balance and Meridional Enthalpy Transports, Rev. Geophys. doi:10.1029/2009RG000323, 2010.

Lucarini, V., Fraedrich, K., and Lunkeit, F.: Thermodynamic analysis of snowball earth hysteresis experiment: Efficiency, entropy production, and irreversibility, Q. J. Roy. Meteorol. Soc., 136, 2-11, 2010.

Myhre, G., Highwood, E. J., Shine, K. P., and Stordal, F.: New estimates of radiative forcing due to well mixed greenhouse gases, Geophys. Res. Lett., 25, 2715-2718, 1998.

Ozawa, H., Ohmura, A., Lorenz, R. D., and Pujol, T.: The second law of thermodynamics and the global climate system: A review of the maximum entropy production principle, Rev. Geophys., 41, 1018, doi:10.1029/2002RG000113, 2003.

Paltridge, G. W.: Climate and thermodynamic systems of maximum dissipation Nature, 279, 630-631, 1979.

Paoletti, S., Rispoli, F., and Sciubba, E.: Calculation of exergetic losses in compact heat exchanger passages, ASME AES, 10, 21 29, 1989.

Pascale, S., Gregory, J. M., Ambaum, M. H. P., and Tailleux, R. G.: Climate entropy budget of the HadCM3 atmosphere-ocean general circulation model and of FAMOUS, its low-resolution version, Clim. Dyn., doi:10.1007/s00382-009-0718-1, 2009.

Peixoto, J. and Oort, A.: Physics of Climate, Springer, New York, 1992.

Ruelle, D.: General linear response formula in statistical mechanics, and the fluctuation-dissipation theorem far from equilibrium, Phys. Lett. A, 245, 220-224, 1998.

Ruelle, D.: A review of linear response theory for general differentiable dynamical systems, Nonlinearity, 22, 855-870, 2009.

Yin, J. F.: A consistent poleward shift of the storm tracks in simulations of 21st century climate, Geophys. Res. Lett., 32, L18701, doi:10.1029/2005GL02368, 2005. 